\title{
Imunidade conferida por vacinas anti-meningocócicas
}

\section{Immunity confered by anti-meningococci vaccines}

\author{
Lucimar Gonçalves Milagres*, Carmo E. A. Melles**
}

\begin{abstract}
MILAGRES, L.G. \& MELLES, C.E.A. Imunidade conferida por vacinas anti-meningocócicas. Rev. Saúde Pública, 27: 221-6, 1993. Em razão da recente epidemia de doença meningocócica causada por $N$. meningitidis B na Grande São Paulo, Brasil, foi feita revisão das epidemias dessa doença ocontidas no Brasil desde o início do século e uma análise das vacinas atuais contra $N$. meningitidis $\mathrm{A}, \mathrm{C}, \mathrm{Y}$ e W135. Também são discutidos os mais recentes avanços no desenvolvimento e aplicação de vacina contra $N$. meningitidis $\mathrm{B}$, um desafio constante para os maiores centros de pesquisa de todo o mundo.
\end{abstract}

Descritores: Neisseria meningitidis, imunologia. Vacinas bacterianas, imunologia. Meningite meningocócica, prevenção.

Neisseria meningitidis ou meningococo é classificada em 12 sorogrupos de acordo com a especificidade imunológica do polissacáride capsular ${ }^{31}$. Diferenças imunológicas entre as principais classes de proteinas da membrana externa são a base para a classificação do microrganismo em sorotipos e subtipos ${ }^{16,20}$, sendo os imunotipos determinados pela especificidade imunológica das moléculas de lipoligossacáride ${ }^{46}$. Desta forma, a designação $N$. meningitidis $\mathrm{B}: 4: \mathrm{P} 1.15: \mathrm{L} 3,7$ indica que a cepa bacteriana pertence ao sorogrupo $B$, sorotipo 4, subtipo P1.15 e imunotipo L3,7.

$N$. meningitidis tem sido, historicamentc, associada a surtos epidêmicos de inflamação das leptomeninges ${ }^{15,16}$. Durante a década de 70, cepas de $N$. meningitidis $\mathrm{B}: 15$ pertencentes a um grupo de clones geneticamente rclacionados, denominado complexo ET-5, emergiram na Europa e causaram surtos epidêmicos na Noruega, Islândia, Dinamarca, Países Baixos e Grã-Bretanha ${ }^{13}$. Caugant e col. ${ }^{13,14}$ (1986, 1991), documentaram a expansão deste clone em diversos continentes, acarretando epidcmias em Cuba (1980), Chile (1985) e Brasil (1988).

No Brasil, a meningite meningocócica foi observada pela prirneira vez em São Paulo no ano de

\footnotetext{
* Seção de Baczeriologia do Instituto Adolfo Lutz. São Paulo, SP - Brasil

** Seção de Bacieriologia do Instituto Adolfo Lutz. São Paulo, SP - Bissil - Coordenador do Centro de Referência Nacional fora Meningites
}

Separatas/Reprints: L.G.Milagres - Av. Dr. Amaldo, 351 - Jarc'im América - 01246-902 - São Paulo, SP - Brasil

Edição subvencionada pela FAPESP. Processo Medicina 93/ 0208-5
1906. A doença manteve-se sob forma endêmica até 1945. Naquele ano teve início uma epidemia que se estendeu até 1951 , sendo que o maior coeficicntc de incidência foi registrado em 1947: 25/ 100.000 habitantes ${ }^{23,34}$. Baseado em relatos de Gomes e col. ${ }^{23}$ e segundo a classificação de Bra$n^{n} a^{8}$ (1953), N. meningitidis sorogrupo A foi o agente responsável por essa epidemia.

A partir de 1971, duas extensas epidemias ocorreram na área metıopolitana da Grande São Paulo, atingindo coeficientes de 100 a 200/100.000 habitantes. A primeira foi causada por $N$. meningitidis $\mathrm{C}$ e a segunda por $N$. meningitidis $\mathrm{A}$. Esta última teve inicio em abril de 1974, quando ainda persistia a primeira $3,27,34$.

Nos primciros meses de 1988, com o número de casos suspcitos de doença meningocócica maior que o espcrado, caracterizou-se uma nova onda epidêmica na Grande São Paulo, causada por $N$. meningitidis $\mathrm{B}: 4: \mathrm{P} 1.15^{40}$. O coeficiente de incidência variou de 4,06 em 1988 a 4,46/100.000 habilantes em 1992 10,11.

Nos últimos meses de 1989 , a situação epidêmica da Grande São Paulo apresentou algumas altcraçōes em relação a doença meningocócica. A proporção de $N$. meningitidis $C$ passou de $9 \%$ nos anos de 1986 a 1988 para 14\% em 1989. Em setcmbro de $1990,46 \%$ dos casos de doença meningocócica diagnosticados laboratorialmente eram devido a $N$. meningitidis $\mathrm{C}$, chegando a $53 \% \mathrm{em}$ outubro ${ }^{10,11}$. O sorotipo 2a predominou entre as amostras de $N$. meningitidis $\mathrm{C}$ isoladas no período de 1976 a 1989. Porém, em meados desse último ano, detectou-se a presença das primeiras cepas de $N$. meningitidis $\mathrm{C}: 2 \mathrm{~b}$ nessa região ${ }^{41}$. Em 1990 , 74\% das cepas de meningococo $C$ pertenciam a este sorotipo, quadro que se manteve até 1992. 
Aproximadamente $70 \%$ dessas cepas pertenciam a um mesmo clone, denominado complexo ET-11, cujo aparecimento foi estreitamente relacionado com a introdução do sorotipo $2 \mathrm{~b}$ no país ${ }^{41}$.

Frente ao conhecimento da antigenicidade e imunogenicidade do meningococo, diferentes vacinas vêm sendo aplicadas com limitados sucessos na prevenção da doença 5,17,24,43,47. Atualmente dispōe-se de vacinas polissacarídicas eficazes em conferir imunidade contra $N$. meningitidis $\mathrm{A}, \mathrm{C}, \mathrm{Y}$ e W13517,21,29. No entanto, crianças menores de 2 anos - a faixa etária de maior risco de aquisição da doença - desenvolvem baixos níveis de anticorpos bactericidas e conseqüentemente apresentam reduzidos níveis de proteção à doença $24,37,45$.

Embora anticorpos bactericidas contra $N$. meningitidis $\mathrm{C}$ tenham sido previamente correlacionados com proteção à doença meningocócica ${ }^{22}$, os estudos relacionados à resposta imune a este agente, em geral se restringem à quantificação de imunoglobulinas e não à análise da atividade funcional dos mesmos. Estudos realizados após a vacinação contra N. meningitidis A, em 1974 e 1975, na Finlândia, estimaram que concentraçð̃es de anticorpos contra o polissacáride A entre 1 e 2 ug/ $\mathrm{ml}$, eram associadas com proteção à doença ${ }^{37}$. Segundo Kayhty e col. ${ }^{28}, 1980$, a duração da imunidade foi dependente da idade e do grau da resposta primária do indivíduo. Crianças menores de 12 meses, que receberam duas doses da vacina, mantiveram níveis protetores de anticorpos por um ano, enquanto estes persistiram por dois anos naquelas de 12 a 17 meses e por um ano em crianças de 18 a 23 meses, ainda que esta última faixa etária tenha recebido uma única dose da vacina.

Segundo Lepow ${ }^{29}$ (1988), a imunização com o polissacáride $C$ de crianças com idade superior a 12 meses resultou em concentraçðes de anticorpos específicos em niveis superiores a $2 \mathrm{ug} / \mathrm{ml}$. Estudo realizado no Estado de São Paulo, durante a epidemia por $N$, meningitidis $\mathrm{C}$, em 1972, demonstrou que tal concentração de anticorpos foi apresentada por $13,6 \%$ a $60 \%$ das crianças de 6 meses a 6 anos $^{36}$. Assim, como o polissacáride A, o grau da resposta imune contra o polissacáride $C$ é diretamente" relacionado a idade do indivíduo ${ }^{21,29} 1,45$.

Embora os títulos iniciais de anticorpos contra os polissacárides $\mathrm{A}$ e $\mathrm{C}$ em crianças imunizadas não sejam significativamente diferentes, Lepow e col. ${ }^{30}, 1977$, observaram que os níveis de anticorpos contra 0 polissacáride $\mathbf{C}$ (anti-C) declinaram mais rapidamente. Durante o primeiro ano após a imunização de indivíduos de 6 a 8 anos, as concentraçōes de anti-A e C diminuíram 32 e $68 \%$, respectivamente. Em estudos posteriores, verificou-se que as tz.sas de declínio foram idênticas para ambos polissacárides ${ }^{30}$. Entre crianças de 2 a 6 anos de idade, detectou-se um declínio de $68 \%$ na concentração de anti-C, 9 meses após a vacinação ${ }^{30}$.

Taunay e col..$^{45}$ avaliaram o efeito protetor da vacina polissacarídica anti-meningococo $\mathrm{C}$ em crianças brasileiras de 6 a 36 meses vacinadas no final de 1972 e acompanhadas clinicamente até meados de 1974 . Os autores relataram que a menor incidência da moléstia em crianças vacinadas, comparada àquelas que receberam um placebo (toxóide diftérico-tetânico), foi significativamente menor apenas para crianças de 24 a 36 meses (3,26 casos por 10.000 indivíduos vacinados contra 7,23 casos no grupo que recebeu o placebo).

A avaliaçāo da resposta imune de crianças de 6 a 12 meses e 13 a 24 meses que receberam duas e uma dose da vacina $A C Y W 135$ demonstrou que somente 40 a $50 \%$ das crianças menores de 18 meses responderam aos polissacárides $\mathrm{Y}$ e W135, contra 80 a $100 \%$ daquelas acima de 18 meses. Houve um rápido declínio desses anticorpos em crianças menores de um ano, sendo que as concentraçð̄es não diferiram daquelas do grupo controle, um ano após a imunização ${ }^{17}$.

Os polissacárides se comportam como antígenos Timo-independentes ( $\mathrm{T}$-independentes), especificamente TI-2, isto é, são fracamente imunogênicos em crianças menores de 2 anos, são desprovidos de atividade policlonal, não induzem memória imunológica, não provocam reaçōes tipo "booster" e os anticorpos formados são de curta duração, além de apresentarem reduzida afinidade ${ }^{1,38}$. Esta natureza é parcialmente atribuída à ausência, nos macrófagos, de determinadas enzimas necessárias para clivagem do carboidrato, tendo como consequiência uma pobre ligação de seus epítopos às glicoproteínas do complexo de histocompatibilidade e reduzida ativação de células $T$. Este fenômeno é particularmente observado em crianças menores de 2 anos ${ }^{1}$. Por outro lado, as subclasses de IgG produzidas pelo sistema imune variam de acordo com a natureza do estímulo antigênico e refletem o mecanismo e a eficácia pelos quais os microrganismos são mortos. Antígenos polissacarídicos induzem, primariamente, a produção de $\operatorname{IgG2}$, enquanto que proteínas estimulam a sintese de IgG1 e IgG3, as quais são mais eficientes na ativação do complemento e na ligação a receptores presentes em neutrófilos e monócitos. IgG2 também apresenta menor capacidade de atravessar a placenta ${ }^{39,44}$.

Os fatores determinantes da imunogenicidade dos polissacárides ainda não estão completamente definidos. Sabe-se da importância do peso molecular do composto, no entanto, pouco se conhece sobre as características físico-químicas do mesmo. 
Estas incluem informaçōes sobre a estrutura terciária e a capacidade de interagir com outras moléculas. Embora o polissacáride $\mathrm{C}$ empregado nas vacinas atuais apresente radicais orto-acetil na sua estrutura, estudos em crianças tem revelado maior imunogenicidade ao carboidrato destituído de tal radical17,50. Portanto, serão ainda necessários maiores conhecimentos sobre os epítopos que induzem anticorpos bactericidas, bem como a melhor forma de apresentação destes para obtenção de vacinas mais eficazes.

Uma das recentes tentativas de aumentar a eficácia das vacinas polissacarídicas consiste na conjugação química do carboidrato a proteínas, processo pelo qual seus epítopos transformam-se em antígenos $T$ dependentes ${ }^{38}$. A vacina de origem cubana (VAMENGOC-BC) foi a primeira, desta natureza, a ser empregada em vacinação em massa, primeiro em Cuba ${ }^{43}$ e a partir de 1989 na Grande São Paulo, São João da Boa Vista (SP), Macapá (AP) e outros Estados do Brasil ${ }^{10,32}$. Na análise da soroconversão contra $N$. meningitidis $\mathrm{C}$ de crianças de 3 a 48 meses, procedentes de São João da Boa Vista e Macapá, não foi detectado aumento significativo nos títulos de anticorpos bactericidas após a vacinação (títulos menores de 1:4). No entanto, niveis significativos destes anticorpos (maiores que 1:64) foram observados em indivíduos de 10 a 14 anos procedentes de São João da Boa Vista*. Estes resultados reforçam a existente correlação entre grau de resposta imune e faixa etária da população vacinada, além de sugerirem que a ligação quím.ica não covalente entre o polissacáride $\mathrm{C}$ e proteínas da membrana externa de $N$. meningitidis foi insuficiente para a transformação do carboidrato em antígeno T-dependente.

Diferente dos demais polissacárides, a resposta imune contra o polissacáride de $N$. meningitidis B caracteriza-se pela produção de IgM, tanto em portadores quanto em pacientes com doença sistêmica ${ }^{31,35}$. Da mesma forma, o polissacáride $B$ purificado e de alto peso molecular induz primariamente à produção de $\operatorname{IgM}^{19,31}$, mesmo quando conjugado a proteínas carreadoras e adsorvidos ao hidróxido de alumínio ${ }^{19,35}$. Esta pobre imunogenicidade é atribuída, principalmente, à semelhança antigênica do polissacáride B com glicolípides e glicoproteínas presentes em células humanas, à sensibilidade a neuraminidases do organismo humano e a instabilidade tridimensional da molécula ${ }^{31}$.

Em vista disto, o desenvolvimento de uma vacina eficaz contra $N$. meningitidis B tem sido uma preocupação de vários centros de pesquisa do

* Dados inéditos. mundo $5,18,43,47$. Em geral, as vacinas produzidas até o momento são constituídas, basicamente, por proteínas da membrana externa, lipoligossacáride residual (LOS), polissacáride $\mathrm{C}$ e adjuvante, geralmente o hidróxido de alumínio ${ }^{5,19,43}$, a maior parte delas conferindo imunidade sorotipo específica ${ }^{17,38,47,49}$. Assim sendo, a grande diversidade antigênica das cepas de $N$. meningitidis B isoladas em determinada área geográfica limita a eficácia dessas vacinas a níveis não aceitáveis para um programa de imunização em massa $5,16,17$.

Os mais recentes estudos de campo objetivando avaliar a eficácia vacinal contra a doença meningocócica causada por $N$. meningitidis $\mathrm{B}$ foram realizados no Chile, Cuba, Noruega e Brasil ${ }^{5,7,33,43}$. $O$ estudo realizado no Chile envolveu indivíduos de 1 a 21 anos de idade e uma vacina composta por proteínas da membrana externa de $N$. meningitidis B:15:P1.3, LOS e polissacáride C. A eficácia encontrada foi de $51 \%$ e após 6 meses os níveis de anticorpos declinaram acentuadamente? A vacina de origem norueguesa demonstrou uma eficácia de 57\% em indivíduos de 13 a 21 anos. $O$ imunógeno era constituído de proteínas da membrana externa de $N$. meningitidis B:15:P1.16, LOS, e hidróxidc de alumínios. Diferente desses resultados, a pesquisa de campo realizada com a vacina cubana, em 7 províncias daquele país, que abrangeu ir.üividuos de 11 a 16 anos, concluiu por uma eficácia vacinal superior a $80 \%{ }^{43}$. Resultados similares foram encontrados na população de 5 meses a 24 anos, vacinadas em Ciego de Avila, Cuba, província com a mais alta taxa de incidência da doença $(30 / 100.000 \text { habitantes })^{12}$. No entanto, estudo caso-controle designado para a avaliação da vacina cubana na Grande São Paulo demonstrou resultados muito diversos àqueles relatados por pesquisadores cubanos. Neste estudo, a eficácia vacinal estimada variou de $-37 \%(-100$ a $73 \%)$, para crianças de 3 a 24 meses, a $74 \%$ (16 a 92\%), para o grupo etário de 4 a 6 anos $^{33}$.

Segundo Sierra e col. ${ }^{43}$ a vacina contra $N$. meningitidis $\mathrm{B}$ e $\mathrm{C}$, de origem cubana, confere proteção à doença causada por diferentes sorotipos de $N$. meningitidis $\mathrm{B}$, proteção esta que seria conferida por proteínas de um "complexo de alto peso molecular", responsável pela indução de anticorpos de reaçס̃es cruzadas.

A quantificação de anticorpos bactericidas tem sido o principal teste laboratorial utilizado na avaliação da imunidade ao meningococo $B$ conferida por vacinação ou doença ${ }^{17,22,26}$. Os estudos laboratoriais da resposta imune de crianças de 3 meses a 6 anos, imunizadas com a vacina cubana na Grande São Paulo,' demonstraram que somente $40 \%$ (22 a 52\%) delas apresentaram aumento mínimo de quatro vezes nos títulos de anticorpos 
bactericidas contra $N$. meningitidis $\mathrm{B}: 4: \mathrm{P} 1.15^{32}$. Os estudos de immunoblot demonstraram que $50 \%$ dos soros com títulos de anticorpos bactericidas maior que 1:4 apresentaram maior reatividade com proteína da membrana externa da classe $1(45 \text { a } 47 \mathrm{Kd})^{32}$. Anticorpos desta natureza induzidos pelas atuais vacinas em testes são direcionados principalmente contra proteínas da membrana externa das classes $1,2,3$ e 5 de $N$. meningitidis ${ }^{17,38,48}$.

Hoiby e col. ${ }^{26}$, quantsticando anticorpos bactericidas, relataram que $98 \%$ dos adultos imunizados com a vacina norueguesa soroconvertcram. Essc estudo também demonstrou a importância da análise da resposta imune de indivíduos pertencentes a diferentes faixas etárias, uma vez que contrariamente ao grau de soroconversão descrito acima, a eficácia vacinal foi estimada em apenas $57 \%$.

Outro parâmetro empregado na avaliação laboratorial da eficácia de uma vacina é a pesquisa de opsoninas-anticorpos que promovem a fagositose do microrganismo. Aumento significativo desses anticorpos foi detectado $\mathrm{em}$ indivíduos vacinados contra $N$. meningitidis $\mathrm{B}: 15: \mathrm{P} 1.16^{25}$.

Estudos de proteção à infecção conferida por imunização passiva têm sido conduzidos em animais, visando identificar çuais os componentes antigênicos de $N$. meningitidis B estariam associados com imunidade e proteção à doença. Em uma dessas investigaçōes, foram utilizados anticorpos monoclonais contra proteínas da classe 1 e 3 . Embora ambos apresentassem atividade bactericida, somente os anticorpos contra proteínas da classe 1 conferiram proteção contra a infecção ${ }^{17}$. Pess:!tados conflitantes foram obtidos por Brodeur e col. ${ }^{9}$ (1985), ao demonstrarem que anticorpos contra proteínas da classe 2, mas não contra aquelas das classes 1 e 5 , induziram proteção à infecção em camundongos. Saukkonen e col. ${ }^{42}$ (1988) por sua vèz, utilizando modelos semelhantes, relataram que anticorpos monoclonais contra LOS preveniram a morte de animais inoculados com N. meningitidis $\mathrm{B}$.

Van der Ley e Poolman ${ }^{47}$ (1991) demonstraram a importância da proteína da classe 1 na indução de anticorpos bactericidas ao imunizarem camundongos com preparaçōes protéicas de $N$. meningitidis B que expressaram proteínas de classe 1 e 3 , somente classe 1 ou 3 e finalmente a combinação de proteínas da classe 1 de duas cepas diferentes. Foi observado que a delcçāo da proteína da classe 3 não altcrou os títulos bactericidas, contrariamente ao ocorrido com proteínas da classe 1. A combinação de diferentes proteínas da classe 1 induziu a produção de anticorpos com reatividade cruzada.

Esses resultados demonstram a dificuldade em se estabelecer a importância de cada componente antigênico da superfície do microrganismo em elucidar anticorpos bactericidas e protetores. Todavia, a maioria dos estudos aponta para a importância da proteína da classe 1 na composição de novas vacinas contra $N$. meningitidis $\mathrm{B}^{17,38,47,50}$.

Recentemente, a importância do ferro na patogênese de contra $N$. meningitidis tem instigado inúmeras pesquisas no sentido de relacionar as proteínas reguladas pelo ferro ( $\mathrm{PRFe}(\mathrm{s})$ ) com imunidade humoral e como prováveis candidatas à produção de vacinas contra $N$. meningitidis $B^{2,4,6,18}$. A presença de anticorpos contra PRFe(s) no soro de pacientes com doença meningocócica indica que estas proteínas são imunogênicas e sintetizadas in vivo ${ }^{2,6}$. Assim, uma das mais recentes alternativas para o desenvolvimento de uma vacina eficaz contra $N$. meningitidis $\mathrm{B}$ é a pesquisa de $\mathrm{PRFe}(\mathrm{s})$ comum à maioria das cepas, antigenicamente estáveis e que induzam anticorpos bactericidas $^{4,18}$. Esta tem sido a linha básica de um projeto de pesquisa interinstitucional, envolvendo os Institutos Adolfo Lutz (SP) e Butantan (SP), Fundação Oswaldo Cruz (RJ) e o Food and Drug Administration (FDA- USA), visando à produção de uma vacina eficaz contra $N$. meningitidis $\mathrm{B}$, desafio constante até o momento.

MILAGRES, L. G. \& MELLES, C. E. A. [Immunity confered by anti-meningococcic vaccines]. Rev. Saúde Pública, 27: 221-6, 1993. In view of a recent epidemic of meningococcal disease caused by serogroup $\mathrm{B} N$. meningitidis in the Greater S. Paulo area (Brazil), a review of the epidemics that occurred in Brazil during the period from 1900 to 1990 is presented. The current status of vaccines against $N$. meningitidis A.C.Y. and W135 is analysed. The recent advances in the development and effectiveness of B. meningococci vaccines are discussed.

Keywords: Neisseriameningitidis, imunology. Bacterial vacinnes, imunology. Meningitis, meningoccoal, prevention.

\section{Referênclas Bibliograficas}

1. ADA, G.L. Modem vaccines: the immunological principles of vaccination. Lancet, 335: 523-6, 1990.

2. ALA'ALDEEN, D.A.; WALL, R.A.; BORRIELO, S.P. IIUman and animal immune response to homologous and heterologous meningococcal 70 kilodalton iron regulated outer membrane protein. In: Atchtman, M. et al. Neis. seria 1990. Berlin, Walter de Gruyter, 1991.p. 177-82.

3. BARATA, R.C.B. Epidemia de doença meningocócica, 1970/1977: aparecimento e disseminação do processo epidêmico. Rev. Saúde Pública, 22: 16-24, 1988.

4. BHATNAGAR, B.N. \& FRASCH, C.E. Expression of Neis. seris meningitidis iron - regulated outer membrane proteins, including a 70-kilodalton transferrin receptor, and their potential for use in vaccines. Infect. Immunol., 58: 2875-81, 1990. 
5. BJUNE, G.; HOIBY, E.E.; GRONNESBY, J.K.; ARNESEN, O.; FREDRIKSEN, J.H.; HALTSTENSEN, A.; HOLTEN, E.; LINDBAK, A.K.; NOKLEBY, H.; ROSENQVIST, E.; SOLBERG, L.K.; CLOSS, O.; FROHOLM, L.O.; LYSTAD, A.; BAKKETEIG, L.S.; HAREID, B. Effect of outer membrane vesicle vaccine against group B meningococcal disease in Norway. Lancet, 338: 1093-6, 1991.

6. BLACK, J.R.; DYER, D.W.; THOMPSON, M.K.; SPARLING, F. Human immune response to iron-repressible outer membrane proteins of Neisseria meningitidis. Infect. Immunol., 54: 710-13, 1986.

7. BOSLEGO, J.; ZOLLINGER, W.; GARCIA, J.; CRUZ, C.; RUIZ, S.; BRANDT, B.; MARTINEZ, M.; ARTHUR, J.; UNDERWOOD, P.; HANKINS, W.; MAYS, J.; GILLY, J.; CHILEAN NATIONAL COMMITTEE FOR MENINGOCOCCAL DISEASE. Eficacy trial of a meningococcal group $B(15: P 1.3)$ outer membrane protein vaccine in Iquique, Chile. In: International Pathogenic Neisseria Conference, 7th. Berlin, 1990. Abstracts. p. 23.

8. BRANHAM, S.E. Serological relationships among meningococci. Bacteriol. Rev., 17: 175-88, 1953.

9. BRODEUR, B.R.; LAROSE, Y.; TSANG, P.; HAMEL, J.; ASHTON, F.; RYAN, A. Protection against infection with Neisseria meningitidis group B serotype $2 b$ by passive immunization with serotype-specific monoclonal antibody. Infect. Immunol., 50: 510-6, 1985.

10. CAMARGO, M.C.C. \& IIIDALGO, N.T.R. Doença meningocócica: a vacina contra o meningococo $B$ e a situação atual na Grande São Paulo. Imunizaçōes, 2: 161-7, 1989.

11. CAMARGO, M.C. \& HIDALGO, N.T.R. A doença meningocócica na Grande São Paulo. Imunizaçōes, 3:4-7, 1990.

12. CAMPA, H.C.; VALCAREL, N.M.; IZOUIERDO, P.L. CIINKARONKO, E.G.; CASANUEVA, G.V. BAR, S.M.; SALAZAR, G.A.; LEGUEN, C.F.; RODRIGUEZ, C.R.; TERRY, M.H.; SIERRA, G.V.G. Efficacy evaluation of the Cuban vaccine "VAMENGOC. BC" against group B Neisseria meningitidis caused disease. In: International Pathogenic Neisseria Conference, 7th, Berlin, 1990. Abstracts. p. 22.

13. CAUGANT, D.A.; FROHOLM, L.O.; BOVRE, K.; HOLTRN, E.; FRASCH, C.E.; MOCCA, L.F.; ZOLLINGER, W.D.; SELANDER, R.K. Intercontinental spread of a genctically distinctive complex of clones of Neisseria meningitidis causing epidemic disease. Proc. Natl. Acad. Sci., 83: 4927-31, 1986.

14. CAUGANT, D.A.; SACCHI, C.T.; FROIIOLM, L.O.; SELANDER, R.K. Genetic structure and epidemiology of scrogroup B Neisseria meningitidis. In: Atchtman, $M$ et al. Neisseria 1990. Berlin, Walter de Gruyter, 1991. p. 37-42.

15. DEVOE, I.W. The meningococcus and mechanisms of pathogenicity. Microbiol. Rev., 46: 162-90, 1982.

16. FRASCH, C.E. Development of meningococcal serotyping. In: Vedros, N.A. Evolution of meningococcal disease. Flórida, CRC Press, 1987. p. 39-54.

17. FRASCH,C.E. Vaccines for prevention of meningococcal disease. Clin. Microbiol. Rev., 2 (Suppl.): 134-8, 1989.

18. FRASCH, C.E.; SACCII, C.T.; BRANDILEONE, M.C.C.; MILAGRES, L.G.; RAMOS, S.R.; VIEIRA, V.S.D. Development of an improved vaccine against group B meningococcal meningitis. Rev. Soc. Bras. Med. Trop., 24: (Suppl): 215-6, 1991.

19. FRASCI, C.E.; ZAHRADNK, J.M.; WANG, L.Y.; MOCCA, L.F.; TSAI, C.M. Antibody response of adults to an aluminum hidroxide-adsorbed Neisseria meningitidis serotype $2 b$ protein-group B polysaccharide vaccine. $J$. Infect. Dis., 158: 710-8, 1988.
20. FRASCH, C.E.; ZOLLINGER, W.D.; POOLMAN, J.T. Serotype antigens of Neisseria meningitidis and a proposed scheme for designation of serotypes. Rev. Infect. Dis., 7: 504-10, 1985.

21. GOLD, R.; LEPOW, M.L.; GOLDSCHNEIDER, I.; GOTSCIILICII, E.C. Immune response of human infants to polysaccharide vaccines of groups $A$ and $C$ Neisseria meningitidis. J. Infect. Dis., 136 (Suppl.): 31-5, 1977.

22. GOLDSCHNEIDER, I.; GOTSCHLICH, E.C.; ARTENSTELN, M.S. Human immunity to the meningococcus. I - The role of humoral antibodies. J. Exp. Med., 129: $1307-26,1969$.

23. GOMES, S.L.; SILVA, M.B.; RIBAS, J.C.; RUGAI, E.; AMOROSINO, A.; CAVE, J.J.D. Meningite cerebrospinal e sulfamidação maciça, preventiva. Rev. Inst. Adolfo Lutz, 10: 77-85, 1950.

24. GRIFFISS, J.M.; BRANDT, B.L.; ALTIERI, P.L.; PIER, G.B.; BERMAN, S.L Safety and immunogenicity of group $Y$ and group W135 meningococcal capsular polysaccharide vaccines in adults. Infect. Immunol., 34: 725-32, 1981.

25. HALSTENSEN, A.; LEHMAN, A.K.; GUTTORMSEN, H.K; VOLLSET, S.E.; BJUNE, G.; NAESS, A. Scrum opsonins to serogroup B meningococci after disease and vaccination. NIPIJ Ann., 14: 157-67, 1991.

26. HOIBY, E.A.; ROSENQVIST, E.; FROHOLM, L.O.; EJUNE, G.; FEIRING, B.; NOKLEBY, H.; RONNILD, E. Bactericidal antibodies after vaccination with the Norwegian meningococcal serogroup B outer membrane vesicle vaccine: a brief survey. NIPH Ann., 14: 147-56, 1991.

27. IVERSSON, L.B. Aspectos epidemiológicos da meningite meningocócica no município de São Paulo (Brasil) no período de 1968 a 1974. Rev. Saúde Pública, 10: 116, 1976.

28. KAYIITY, H.; KARANKO, V.; PELTOLA, H.; SARNA, S.; MAKELA, P.H. Serum antibodies to capsular polysaccharide vaccine of group A Neisseria meningitidis followed for three years in infants and children. $J . I n$. fect. Dis., 142: 861-68, 1980.

29. LEPOW, M.L. Meningococcal vaccines. In: Plopkin, S.A. \& Mortimer, E.A. Vaccines. Philadelphia, W.B. Sauders, 1988. p. 263-70.

30. LEPOW, M.L.; GOLDSCINEIDER, I.; GOLD, R.; RANDOLPH, M.; GOTSCHLICH, E.C. Persistence of antibody following immunization of children with groups $A$ and $C$ meningococcal polysaccharide vaccines. Pedialrics, 60: 673-80, 1977.

31. LIFTELY, M.R.; MORTNO, C.; LINDON, J.C. An integrated molecular and immunological approach towards a meningococcal group B vaccine. Vaccine, 5: 11-26, 1987.

32. MILAGRES, L.G. Avaliação da resposta imune em crianças de 3 a 83 meses de idade vacinadas contra Neisseria meningitidis B e C. São Paulo, 1992. [Dissertação de Mestrado - Faculdade de Ciências Farmacêuticas da USP].

33. MORAES, J.C.; PERKINS, B.A.; CAMARGO, M.C.C.; IIIDAL.GO, N.T.R.; BARBOSA, II.A.; SACCHI, C.T.; GRAL, I.M.L.; GATTAS, V.L.; VASCONCELOS, H.G.; PLIKAYTIS, B.D.; WENGER, J.D.; BROOME, C.V. Protective efficacy of a scrogroup B meningococcal vaccinc in São Paulo, Brazil. Lancet, 340: 1074$78,1992$.

34. MORAIS, J.S.; MUNFORD, R.S.; RISI, J.B.; ANTEZANA, E.; FELDMAN, R.A. Epidemic disease due to serogroup C Neisseria meningitidis in São Paulo, Brazil. J.Infect. Dis., 129: 568-71, 1974. 
35. MORENO, C.; LIFELY, M.R.; ESDAILE, J. Effect of aluminum ions on chemical and immunological properties of meningococcal group B polysaccharide. Infect. Immunol., 49: 587-92, 1985 .

36. NETO, V.A.; FINGER, H.; GOTSCHLICH, E.C.; FELDMAN, R.A.; AVILA, C.A.; KONISHI, S.R.; LAUS, W.C. Serologic response to serogroup $C$ meningococcal vaccine in Brazilian preschool children. Rev. Inst. Med. Trop., 16: 149-53, 1974

37. PELTOLA, H.; MAKELA, P.H.; KAYHTY, H.; JOUSIMIES, H.; HERVA, E.; HALLSTROM, K.; SIVONEM, A.; RENKONEN, O.V.; PETTAY, O.; KARANKO, V.; AHVONEN, P.; SARNA, S. Clinical efficacy of meningococcus group A capsular polysaccharide vaccine in children three months to five years of age. $N$. Engl.J. Med., 297: 686-91, 1977.

38. POOLMAN, J.T, Polysaccharides and membrane vaccines. In: Nizrahi, A. Bacterial vaccines. New York, WileyLiss, 1990. p. 57-86.

39. ROBBINS, J.B. Vaccines for the prevention of encapsulated bacterial diseases: current staius, problems and prospects for the future. Immunochemistry, 15: 839-54, 1978.

40. SACCHI, C.T.; PESSOA, L.L.; RAMOS, S.R.; MILAGRES, L.G.; CAMARGO, M.C.; HIDALGO, N.T.; MELLES, C.E.A.; CAUGANT, D.A.; FRASCH, C.E. Ongoing group B Neisseria meningitidis epidemic in Saao Paulo, Brazil, due to increased prevalence of a single clone of the ET-5 complex. J. Clin. Microbiol., 30: 1734-38, 1992.

41. SACCHI, C.T.; ZANELLA., R.C.; CAUGANT, D.A.; FRASCH, C.E.; HIDALGO, N.T.; MILAGRES, LG.; PESSOA, L.L.; RAMOS, S.R.; CAMARGO, M.C.; MELLES, C.E.A. Emergence of a new clone of serogroup C Neisseria meningitidis in São Paulo, Brazil. $J$. Clin. Microbiol., 30: 1282-86, 1992.

42. SAUKKONEN, K.; LEDNONEN, M.; KAYHTY, H.; ABDILLAHI, H.; POOLMAN, J.T. Monoclonal antibodies to the rough lipopolysaccharide of Neisseria meningitidis protect infant rats from meningococcal infection. $J$. Infect. Dis, 158: 209-12, 1988.

43. SIERRA, G. V.G.; CAMPA, H.C.C.; GARCIA, I.L.; SOTOLONGO, P.F.; IZQUIERDO, P.L.; VALCARCEL,
N.M.; CASANUEVA, G.V.; BARO, M.S.; LEGUEN, C.F.; RODRIGUEZ, C.R.; TERRY, M.H. Efficacy evaluation of the Cuban vaccine VA-MENGOC-BCR against disease caused by serogroup B Neisseria meningitidis. In: Atchtman, M. et al. Neisseria 1990. Berlin, Walter de Gruyter, 1991. p. 129-34.

44. SJUREN, H.; WEDEGE, E.; ROSENQVIST, E.; NAESS, A.; HALSTENSEN, A.; MATRE, R.; SOLBERG, C.O. IgG subclass antibodies to serogroup $B$ meningococcal outer membrane antigens following infection and vaccination. Acta Pathol. Microbiol. Scand., 98: $1061-69,1990$.

45. TAUNAY, A.E.; FELDMAN, R.A.; BASTOS, C.O.; GALVẢO, P.A.A.; MORAIS, J.S.; CASTRO, I.O. Avaliação do efeito protetor de vacina polissacarídica antimeningocócica do grupo $\mathrm{C}$, em crianças de 6 a 36 meses. Rev. Inst. Adolfo Lutz, 38: 77-82, 1978.

46. TSAI, C.M.; MOCCA, L.F.; FRASCH, C.E. Immunotype epitopes of Neisseria meningitidis lipooligosaccharide types 1 through 8. Infect. Immunol., 55: 1652-6, 1987.

47. Van der LEY, P. \& POOLMAN, J.T. The class 1 outer membrane protein of Neisseria meningitidis: prediction of topology and construction of a multivalent vaccine strain. In: Atchtman, M. et al. Neisseria 1990. Berlin, Walter de Gruyter, 1991. p. 296-300.

48. WEDEGE, E. \& FROHOLM, L.O. Human antibody response to a group B serotype 2 a meningococcal vaccine determined by immunobloting. Infect. Immunol., 51: $571-8,1986$

49. ZOLLINGER, W.D.; BOSLEGO, J.; MORAM, E.E.; BRANDT, B.; COLLINS, H.; MANDRELL, R.; ALTIERE, P.; BERMAN, S. Bactericidal antibody response to a polyvalent meningococcal proteinpolysaccharide vaccine. In: Robbins, J.B. Bacterial vaccines. New York, Praeger Publications, 1987. p. $245-61$.

50. ZOLLINGER, W.D. Meningococcal meningitidis. In: Stanley J. Crys, Jr. Vaccines and Immunotherapy. New York, Pergamon Press, 1991. p. 113-26.

Recebido para publicaçäo em 3.9.1992 Aprovado para publicação em 8.3.1993 